\title{
ON A GENERAL RATIO ERGODIC THEOREM WITH WEIGHTED AVERAGES
}

\author{
RYOTARO SATO
}

\begin{abstract}
A general ratio ergodic theorem with weighted averages is shown by utilizing a method of R. V. Chacon. The theorem contains Chacon's general ergodic theorem as a special case.
\end{abstract}

Let $(X, \mathscr{M}, m)$ be a $\sigma$-finite measure space and let $T$ be a linear contraction on $L^{1}(m)$. Let $\left\{w_{n} ; n \geqq 1\right\}$ be a sequence of nonnegative numbers whose sum is one, and let $\left\{u_{n} ; n \geqq 0\right\}$ be the sequence defined by

$$
u_{n}=w_{1} u_{n-1}+\cdots+w_{n} u_{0}, \quad u_{0}=1 .
$$

In this note we shall show the following

THEOREM. If $\left\{p_{n} ; n \geqq 0\right\}$ is a sequence of nonnegative measurable functions with $|T g| \leqq p_{n+1}$ whenever $g \in L^{1}(m)$ and $|g| \leqq p_{n}$ then for any $f \in L^{1}(m)$,

$$
\lim _{n \rightarrow \infty} \sum_{k=0}^{n} u_{k} T^{k} f(x) / \sum_{k=0}^{n} u_{k} p_{k}(x)
$$

exists and is finite a.e. on $\left\{x \mid \sum_{k=0}^{\infty} u_{k} p_{k}(x)>0\right\}$.

Proof. Let $I$ be the positive integers, $\Sigma$ all possible subsets, and $\mu$ the measure on $(I, \Sigma)$ defined by $\mu(\{1\})=1$ and $\mu(\{i\})=1-w_{1}-\cdots-w_{i-1}$ for $i \geqq 2$. Let $\left\{\beta_{n} ; n \geqq 1\right\}$ be the sequence defined by

$$
\beta_{n}=w_{n} /\left(1-w_{1}-\cdots-w_{n-1}\right),
$$

$\beta_{1}=w_{1}$. Let $S$ he the linear operator on $L^{1}(\mu)$ satisfying $S h_{1}=\sum_{n=1}^{\infty} \beta_{n} h_{n}$ and $S h_{i}=\left(1-\beta_{i-1}\right) h_{i-1}$ for $i \geqq 2$, where $h_{n}$ denotes the indicator function of the set $\{n\}$. Then it is known (cf. [2]) that $\|S\|=1$ and $S^{n} h_{1}(1)=u_{n}$ for each $n \geqq 0$. Thus the direct product $S \times T$ of $S$ and $T$ is a linear contraction on $L^{1}(\mu \times m)$ and satisfies $(S \times T)^{n} h_{1} f(1, x)=S^{n} h_{1}(1) T^{n} f(x)=u_{n} T^{n} f(x)$. Now define a sequence $\left\{\tilde{p}_{n} ; n \geqq 0\right\}$ of nonnegative measurable functions on $\left(I \times X, \sum \cdots \mathscr{M}, \mu \times m\right)$ by $\tilde{p}_{n}(i, x)=S^{n} h_{1}(i) p_{n}(x)$. It is easily checked that

Received by the editors December 3, 1971.

AMS 1970 subject classifications. Primary 47A35.

Key words and phrases. Linear contraction, Chacon's ergodic theorem. 
$|(S \times T) \tilde{g}| \leqq \tilde{p}_{n+1}$ whenever $\tilde{g} \in L^{1}(\mu \times m)$ and $|\tilde{g}| \leqq \tilde{p}_{n}$. Hence Chacon's ergodic theorem [1] completes the proof of the theorem.

\section{BIBLIOGRAPHY}

1. R. V. Chacon, Operator averages, Bull. Amer. Math. Soc. 68 (1962), 351-353. MR 26 \#287.

2. - Ordinary means imply recurrent means, Bull. Amer. Math. Soc. 70 (1964), 796-797. MR 29 \#5977.

Josai University, Sakado, Saitama 350-02, Japan 